\title{
FINITE ORDER SOLUTIONS OF SECOND ORDER LINEAR DIFFERENTIAL EQUATIONS
}

\author{
GARY G. GUNDERSEN
}

\begin{abstract}
We consider the differential equation $f^{\prime \prime}+A(z) f^{\prime}+B(z) f=0$ where $A(z)$ and $B(z)$ are entire functions. We will find conditions on $A(z)$ and $B(z)$ which will guarantee that every solution $f \not \equiv 0$ of the equation will have infinite order. We will also find conditions on $A(z)$ and $B(z)$ which will guarantee that any finite order solution $f \not \equiv 0$ of the equation will not have zero as a Borel exceptional value. We will also show that if $A(z)$ and $B(z)$ satisfy certain growth conditions, then any finite order solution of the equation will satisfy certain other growth conditions. Related results are also proven. Several examples are given to complement the theory.
\end{abstract}

$\therefore$

1. Introduction. Consider the second order linear differential equation

$$
f^{\prime \prime}+A(z) f^{\prime}+B(z) f=0
$$

where $A(z)$ and $B(z) \not \equiv 0$ are entire functions, and where $A(z)$ is transcendental. It is well known that each solution $f$ of equation (1.1) is an entire function, and that if $f_{1}$ and $f_{2}$ are any two linearly independent solutions of (1.1), then at least one of $f_{1}, f_{2}$ must have infinite order (see [11 or 16, pp. 167-168]). Hence, "most" solutions of (1.1) will have infinite order. On the other hand, there are some equations of the form (1.1) that possess a solution $f \not \equiv 0$ of finite order; for example, $f(z)=e^{-z}$ satisfies $f^{\prime \prime}+e^{z} f^{\prime}+\left(e^{z}-1\right) f=0$.

Thus a natural question is: what conditions on $A(z)$ and $B(z)$ will guarantee that every solution $f \not \equiv 0$ of (1.1) has infinite order? In this paper we will exhibit several general classes of equations of the form (1.1) that have the property that each equation in each class possesses only nontrivial solutions of infinite order.

We mention that if $P(z)$ and $Q(z)$ are polynomials, then [23, p. 108] every solution $f$ of the equation

$$
f^{\prime \prime}+P(z) f^{\prime}+Q(z) f=0
$$

has finite order. We also note that if $A(z)$ is a polynomial and $B(z)$ is a transcendental entire function, then every solution $f \not \equiv 0$ of $f^{\prime \prime}+A(z) f^{\prime}+B(z) f=0$ has infinite order (see [11] or Corollary 1 in $\S 2$ ).

Two related questions to the above question are: (i) If an equation of the form (1.1) possesses a solution $f \not \equiv 0$ of finite order, then how do the properties of $A(z)$ and $B(z)$ affect the properties of $f$ ? (ii) What conditions on $A(z)$ and $B(z)$ in $(1.1)$

Received by the editors December 16, 1986.

1980 Mathematics Subject Classification (1985 Revision). Primary 34A20, 30D35; Secondary 34C11, 34C10, 34A30.

Key words and phrases. Linear differential equation, entire function, finite order of growth, exponent of convergence of the zero-sequence. 
(or on $P(z)$ and $Q(z)$ in (1.2)) will guarantee that any finite order solution $f \not \equiv 0$ of (1.1) (or (1.2)) will satisfy the condition that the exponent of convergence of the zero-sequence of $f$ equals the order of $f$ ? We will prove some results concerning these two questions, plus we will prove some related results.

To complement our results, we shall give many examples of equations (1.1) and (1.2).

2. Statement of the main results. For an entire function $w(z)$, we will let $\rho(w)$ denote the order of $w$, and when $w \not \equiv 0$ we will let $\lambda(w)$ denote the exponent of convergence of the zero-sequence of $w$. We will assume that the reader is familiar with the fundamental results and the standard notations of R. Nevanlinna's theory of meromorphic functions (see [15]).

We consider the differential equation

$$
f^{\prime \prime}+A(z) f^{\prime}+B(z) f=0
$$

where $A(z)$ and $B(z) \not \equiv 0$ are entire functions.

Our first result shows, among other things, that if $f \not \equiv 0$ is a finite order solution of equation (2.1) where $\rho(A) \neq \rho(B)$, then $\lambda(f)=\rho(f)$.

THEOREM 1. If $f \not \equiv 0$ is a solution of equation (2.1) where $\rho(f)<\infty$, then exactly one of the following four cases must occur:

(i) $\lambda(f)=\rho(f)$.

(ii) $\lambda(f)<\rho(f)$, and $A(z)$ and $B(z)$ are both transcendental with $\rho(A)=\rho(B)$. Furthermore, $T(r, A)=T(r, B)+O(\log r)$ as $r \rightarrow \infty$ outside a set $E$ that has finite logarithmic measure.

(iii) $1 \leq \lambda(f)<\rho(f)$, and $A(z)$ and $B(z)$ are both nonconstant polynomials that satisfy $\operatorname{deg}(B)=2 \operatorname{deg}(A)>\operatorname{deg}\left(4 B-A^{2}\right)$ and $\rho(f)=1+\operatorname{deg}(A)$.

(iv) $f$ has only finitely many zeros, $\rho(f) \geq 1$, and $A(z)$ and $B(z)$ are both polynomials. Furthermore, we must have exactly one of the following four situations:

(a) $\operatorname{deg}(B)>2 \operatorname{deg}(A)$ and $\rho(f)=1+(\operatorname{deg}(B)) / 2$.

(b) $\operatorname{deg}(B)=2 \operatorname{deg}(A)$ and $\rho(f)=1+\operatorname{deg}(A)$.

(c) $\operatorname{deg}(B)<2 \operatorname{deg}(A)$ and $\rho(f)=1+\operatorname{deg}(B)-\operatorname{deg}(A)$.

(d) $\operatorname{deg}(B)<2 \operatorname{deg}(A)$ and $\rho(f)=1+\operatorname{deg}(A)$.

All cases in Theorem 1 can occur. For any $\alpha$ where $0 \leq \alpha \leq \infty$, case (ii) in Theorem 1 can occur with $\rho(A)=\rho(B)=\alpha$, because if $H(z)$ is any entire function and $k$ is any positive integer, then $f(z)=\exp \left(z^{k}\right)$ satisfies the equation

$$
f^{\prime \prime}+\left(H(z)-k z^{k-1}\right) f^{\prime}-\left(k(k-1) z^{k-2}+k z^{k-1} H(z)\right) f=0 .
$$

We note that $f_{1}(z)=(\sin z) \exp \left(z^{2} / 2\right)$ and $f_{2}(z)=(\cos z) \exp \left(z^{2} / 2\right)$ both satisfy the equation $f^{\prime \prime}-2 z f^{\prime}+z^{2} f=0$; hence case (iii) in Theorem 1 can occur. For a more general example of Theorem 1(iii), see Example 4 in $\S 11$. Situations (a)-(d) in case (iv) of Theorem 1 can occur by Example 5 in $\S 11$. There are many examples of case (i) in Theorem 1; e.g. $f(z)=e^{z}+1$ satisfies $f^{\prime \prime}+e^{z} f^{\prime}-e^{z} f=0$.

Theorem 1 gives an extension of the following result of Bank and Laine: If $f \not \equiv 0$ is a solution of $f^{\prime \prime}+Q(z) f=0$ where $Q(z)$ is a nonconstant polynomial, and if $\lambda(f)<\rho(f)$, then $f$ has only finitely many zeros (see [4, Theorem 1]). Regarding cases (iii) and (iv) of Theorem 1, we mention the work of Pöschl [20] and Wittich (see $[24$, pp. 69-70]). 
I do not know whether we need the exceptional set $E$ in case (ii) of Theorem 1 .

We mention that if $\alpha$ is any given real number that satisfies $0 \leq \alpha \leq 1$, then there exist three transcendental entire functions $A, B$, and $f$, all of finite order, such that $f^{\prime \prime}+A(z) f^{\prime}+B(z) f=0$ and $\delta(0, f)=\alpha$ (see Example 3 in $\left.\S 11\right)$.

It is very easy to prove the following result.

THEOREM 2. If $f \neq 0$ is a solution of equation (2.1) where $\rho(f)<\infty$, then as $r \rightarrow \infty$,

$$
T(r, B) \leq T(r, A)+O(\log r) .
$$

COROLlary 1. Let $A(z)$ and $B(z)$ be entire functions where either (i) $\rho(A)<$ $\rho(B)$, or (ii) $A$ is a polynomial and $B$ is transcendental. Then every solution $f \not \equiv$ of (2.1) has infinite order.

Frei [11] proved Corollary 1(ii).

Our next result shows that if $f \not \equiv 0$ is a finite order solution of (2.1) where the growth of $A(z)$ dominates the growth of $B(z)$ in some angle, then $f$ will satisfy certain growth conditions in the angle.

THEOREM 3. Let $A(z)$ and $B(z) \not \equiv 0$ be entire functions such that for real constants $\alpha, \beta, \theta_{1}, \theta_{2}$, where $\alpha>0, \beta>0$, and $\theta_{1}<\theta_{2}$, we have

$$
|A(z)| \geq \exp \left\{(1+o(1)) \alpha|z|^{\beta}\right\}
$$

and

$$
|B(z)| \leq \exp \left\{o(1)|z|^{\beta}\right\}
$$

as $z \rightarrow \infty$ in $\theta_{1} \leq \arg z \leq \theta_{2}$. Let $\varepsilon>0$ be a given small constant, and let $S(\varepsilon)$ denote the angle $\theta_{1}+\varepsilon \leq \arg z \leq \theta_{2}-\varepsilon$.

If $f \not \equiv 0$ is a solution of equation (2.1) where $\rho(f)<\infty$, then the following conclusions hold:

(i) There exists a constant $b \neq 0$ such that $f(z) \rightarrow b$ as $z \rightarrow \infty$ in $S(\varepsilon)$. Furthermore,

$$
|f(z)-b| \leq \exp \left\{-(1+o(1)) \alpha|z|^{\beta}\right\}
$$

as $z \rightarrow \infty$ in $S(\varepsilon)$.

(ii) For each integer $k \geq 1$,

$$
\left|f^{(k)}(z)\right| \leq \exp \left\{-(1+o(1)) \alpha|z|^{\beta}\right\}
$$

as $z \rightarrow \infty$ in $S(\varepsilon)$.

Theorem 3 generalizes and improves some results of Ozawa (see [19, Theorem 2]) who considered a similar hypothesis to Theorem 3 except that he made the additional assumption that $B(z)$ is a polynomial. An illustration of Theorem 3 is: for any constant $b \neq 0, f(z)=e^{z}+b$ satisfies

$$
f^{\prime \prime}+\left(e^{z}-b e^{-z}+b-2\right) f^{\prime}+\left(1-e^{z}\right) f=0 .
$$

This example shows that the inequalities (2.6) and (2.7) are both sharp in the sense that neither of the two numbers " $\alpha$ " or " $\beta$ " in either (2.6) or (2.7) could be replaced by a larger number. For more examples of Theorem 3 where (2.6) and (2.7) are also sharp in this sense, see Example 2 in $§ 11$. 
Even though the coefficients of $f$ and $f^{\prime}$ in (2.8) have the same order, it turns out that if we switch these coefficients, then every nontrivial solution of the resulting equation will have infinite order, i.e., every solution $f \not \equiv 0$ of

$$
f^{\prime \prime}+\left(1-e^{z}\right) f^{\prime}+\left(e^{z}-b e^{-z}+b-2\right) f=0
$$

(where $b \neq 0$ is a constant) has infinite order. This follows from the next result, which can be contrasted with Theorem 3 .

THEOREM 4. Let $A(z)$ and $B(z)$ be entire functions such that for real constants $\alpha, \beta, \theta_{1}, \theta_{2}$ where $\alpha>0, \beta>0$, and $\theta_{1}<\theta_{2}$, we have

$$
|B(z)| \geq \exp \left\{(1+o(1)) \alpha|z|^{\beta}\right\}
$$

and

$$
|A(z)| \leq \exp \left\{o(1)|z|^{\beta}\right\}
$$

as $z \rightarrow \infty$ in $\theta_{1} \leq \arg z \leq \theta_{2}$. Then every solution $f \not \equiv 0$ of (2.1) has infinite order.

Although Theorem 4 and Corollary 1 can be compared, there are differential equations to which we can apply Theorem 4 but not Corollary 1. For example, every solution $f \not \equiv 0$ of either (2.9) or $f^{\prime \prime}+\exp (P(z)) f^{\prime}+\exp (-P(z)) f=0$ (where $P(z)$ is a nonconstant polynomial) is of infinite order.

By combining Theorem 3 and the classical Phragmén-Lindelöf theorem, we will easily obtain the following result.

THEOREM 5. Let $A(z)$ and $B(z) \not \equiv 0$ be entire functions, and let $\alpha>0$ and $\beta>0$ be constants where $\rho(B)<\beta$. Suppose that for any given $\varepsilon>0$, there exist two finite collections of real numbers $\left\{\phi_{k}\right\}$ and $\left\{\theta_{k}\right\}$ that satisfy $\phi_{1}<\theta_{1}<\phi_{2}<$ $\theta_{2}<\cdots<\phi_{n}<\theta_{n}<\phi_{n+1}$ where $\phi_{n+1}=\phi_{1}+2 \pi$, and

$$
\sum_{k=1}^{n}\left(\phi_{k+1}-\theta_{k}\right)<\varepsilon
$$

such that

$$
|A(z)| \geq \exp \left\{(1+o(1)) \alpha|z|^{\beta}\right\}
$$

as $z \rightarrow \infty$ in $\phi_{k} \leq \arg z \leq \theta_{k}(k=1,2, \ldots, n)$.

Then every solution $f \not \equiv 0$ of equation (2.1) has infinite order.

Ozawa [19, Theorem 3] proved Theorem 5 under the additional hypothesis that $B(z)$ is a polynomial. It follows from Theorem 5 that if $B(z) \not \equiv 0$ is an entire function and $n \geq 1$ is an integer, then every solution $f \not \equiv 0$ of the following two equations has infinite order:

(i) $f^{\prime \prime}+\sin \left(z^{n}\right) f^{\prime}+B(z) f=0 \quad$ where $\rho(B)<n$;

(ii) $f^{\prime \prime}+\cos \left(z^{n / 2}\right) f^{\prime}+B(z) f=0$ where $\rho(B)<n / 2$.

Furthermore, given any real number $\mu>1$, the Mittag-Leffler function can be used to construct an entire function $A(z)$ with $\rho(A)=\mu$ such that if $B(z) \not \equiv 0$ is entire with $\rho(B)<\mu$, then the hypothesis of Theorem 5 will be satisfied (see [10, p. 50]).

From Theorem 2 we see that if an equation of the form (2.1) possesses a solution $f \not \equiv 0$ of finite order, then it is necessary that $\rho(B) \leq \rho(A)$. Given any $\alpha$ where $0 \leq \alpha \leq \infty$, there exist such examples where $\rho(B)=\rho(A)=\alpha$ (see (2.2)). Also, 
given any integer $n \geq 1$, there exist such examples where $\rho(B)<\rho(A)=n$ (see Examples 1 and 2 in $\S 11$ ). Thus a natural question is: If $f \not \equiv 0$ is a solution of an equation of the form (2.1) where $\rho(B)<\rho(A)<\infty$ and where $\rho(A)$ is not a positive integer, then is it necessarily true that $\rho(f)=\infty$ ? A partial result on this question is given by the next result.

THEOREM 6. If $f \not \equiv 0$ is a solution of (2.1) where either (i) $\rho(B)<\rho(A)<$ $1 / 2$, or (ii) $A(z)$ is transcendental with $\rho(A)=0$ and $B(z)$ is a polynomial, then $\rho(f)=\infty$.

Ozawa [19, Theorem 1] proved that Theorem 6 holds under the additional hypothesis that $B(z)$ is a polynomial.

Several authors have studied the question of whether the particular differential equation

$$
f^{\prime \prime}+e^{-z} f^{\prime}+B(z) f=0
$$

(where $B(z) \not \equiv 0$ is entire) can possess a solution $f \not \equiv 0$ of finite order. Frei [12] showed that if $B(z) \equiv C$ where $C \neq 0$ is a constant, then equation (2.14) will possess a solution $f \not \equiv 0$ of finite order if and only if $C=-n^{2}$ where $n$ is a positive integer (and in this case $f(z)$ is a polynomial in $e^{z}$ of degree $n$ ). Other proofs of Frei's result can be found in $[\mathbf{1 9}, \mathbf{2 5}]$, and it might be mentioned that Wittich $[\mathbf{2 5}]$ showed that if $f \not \equiv 0$ is a finite order solution of $f^{\prime \prime}+P_{1}\left(e^{z}\right) f^{\prime}+P_{2}\left(e^{z}\right) f=0$ where $P_{1}(z)$ and $P_{2}(z) \not \equiv 0$ are polynomials, then $f(z)=e^{b z} Q\left(e^{z}\right)$ where $Q(z)$ is a polynomial and $b$ is a constant. A generalization of Frei's result was proven by Bank and Laine [5] in their investigation into which equations of the form (2.14) (where $B(z)$ is a nonzero constant) can possess a solution $f \not \equiv 0$ where $\lambda(f)<\infty$. By completing results of Ozawa [19], Amemiya and Ozawa [1], and the author [13], Langley [17] has shown that if $B(z)$ is a nonconstant polynomial, then every solution $f \not \equiv 0$ of equation (2.14) has infinite order. The author [13, Theorem 1] showed that if $B(z)$ is transcendental entire with $\rho(B) \neq 1$, then every solution $f \not \equiv 0$ of $(2.14)$ is of infinite order. The case when $\rho(B)=1$ is exceptional, since $f(z)=e^{z}$ satisfies $f^{\prime \prime}+e^{-z} f^{\prime}-\left(1+e^{-z}\right) f=0$. The next result will give a generalization of $[\mathbf{1 3}$, Theorem 1].

THEOREM 7. Let $\left\{\phi_{k}\right\}$ and $\left\{\theta_{k}\right\}$ be two finite collections of real numbers that satisfy $\phi_{1}<\theta_{1}<\phi_{2}<\theta_{2}<\cdots<\phi_{n}<\theta_{n}<\phi_{n+1}$ where $\phi_{n+1}=\phi_{1}+2 \pi$, and set

$$
\mu=\max _{1 \leq k \leq n}\left(\phi_{k+1}-\theta_{k}\right) \text {. }
$$

Suppose that $A(z)$ and $B(z)$ are entire functions such that for some constant $\alpha \geq 0$,

$$
|A(z)|=O\left(|z|^{\alpha}\right)
$$

as $z \rightarrow \infty$ in $\phi_{k} \leq \arg z \leq \theta_{k}$ for $k=1, \ldots, n$, and where $B(z)$ is transcendental with $\rho(B)<\pi / \mu$.

Then every solution $f \not \equiv 0$ of equation (2.1) has infinite order.

There are many examples of Theorem 7 , and we mention the following two:

(i) Let $Q(z)$ be a nonconstant polynomial, let $h(z) \not \equiv 0$ be entire with $\rho(h)<$ $\operatorname{deg}(Q)$, and let $B(z)$ be transcendental entire with $\rho(B) \neq \operatorname{deg}(Q)$. Then from Theorem 7 and Corollary 1 , it follows that every solution $f \not \equiv 0$ of the equation

$$
f^{\prime \prime}+h(z) e^{Q(z)} f^{\prime}+B(z) f=0
$$

has infinite order. This generalizes $[\mathbf{1 3}$, Theorem 1]. 
(ii) If $B(z)$ is transcendental entire with $\rho(B)<4 / 3$, then from Theorem 7 , every solution $f \not \equiv 0$ of the equation $f^{\prime \prime}+\left\{\exp \left(z^{2}\right)+\exp \left(i z^{2}\right)\right\} f^{\prime}+B(z) f=0$ has infinite order.

Next, we make the following observation. Even though at least one of every two linearly independent solutions of equation (1.1) must have infinite order, it is possible for an equation of the form (1.1) to possess two linearly independent solutions $f_{1}$ and $f_{2}$ where $\rho\left(f_{1}\right)<\infty$ and $\lambda\left(f_{2}\right)<\infty$; for example, if $b \neq 0$ is a constant, then $f_{1}(z)=e^{z}$ and $f_{2}(z)=\exp \left\{z+b e^{-2 z}\right\}$ both satisfy the equation

$$
f^{\prime \prime}+2 b e^{-2 z} f^{\prime}-\left(1+2 b e^{-2 z}\right) f=0 .
$$

Other examples of this kind can be found in a result of Bank and Laine [5, Theorem 2].

Last, we mention that there have been several recent results that concern the question of whether an equation of the form $f^{\prime \prime}+G(z) f=0$ where $G(z)$ is transcendental entire, can possess either (i) a solution $f \not \equiv 0$ where $\lambda(f)<\infty$, or (ii) two linearly independent solutions $f_{1}$ and $f_{2}$ where $\lambda\left(f_{1}\right)<\infty$ and $\lambda\left(f_{2}\right)<\infty$ ? See, for example, $[\mathbf{3}, \mathbf{4}, \mathbf{5}, \mathbf{6}, \mathbf{7}, \mathbf{8}, \mathbf{1 7}, \mathbf{2 1}, \mathbf{2 2}]$.

This paper is organized as follows. In $\S 3$ we give some lemmas which will be used in the proofs of Theorems 1-7, while in $\S \S 4-10$ we will prove Theorems 1-7. In $\S 11$ we will give some more examples of equations of the form (2.1) which will further exhibit the sharpness of some of our results, and which will also illustrate some possibilities that can occur.

3. Some lemmas. We shall use Lemmas 1-6 below in the proofs of our results.

LEMMA 1 [14]. Let $w$ be a transcendental entire function of finite order $\rho$, let $\Gamma=\left\{\left(k_{1}, j_{1}\right),\left(k_{2}, j_{2}\right), \ldots,\left(k_{m}, j_{m}\right)\right\}$ denote a finite set of distinct pairs of integers that satisfy $k_{i}>j_{i} \geq 0$ for $i=1, \ldots, m$, and let $\varepsilon>0$ be a given constant. Then the following three statements hold:

(i) There exists a set $E_{1} \subset[0,2 \pi)$ that has linear measure zero, such that if $\psi_{0} \in[0,2 \pi)-E_{1}$, then there is a constant $R_{0}=R_{0}\left(\psi_{0}\right)>0$ so that for all $z$ satisfying $\arg z=\psi_{0}$ and $|z| \geq R_{0}$, and for all $(k, j) \in \Gamma$, we have

$$
\left|w^{(k)}(z) / w^{(j)}(z)\right| \leq|z|^{(k-j)(\rho-1+\varepsilon)} .
$$

(ii) There exists a set $E_{2} \subset(1, \infty)$ that has finite logarithmic measure, such that for all $z$ satisfying $|z| \notin E_{2} \cup[0,1]$ and for all $(k, j) \in \Gamma$, the inequality $(3.1)$ holds.

(iii) There exists a set $E_{3} \subset[0, \infty)$ that has finite linear measure, such that for all $z$ satisfying $|z| \notin E_{3}$ and for all $(k, j) \in \Gamma$, we have

$$
\left|w^{(k)}(z) / w^{(j)}(z)\right| \leq|z|^{(k-j)(\rho+\varepsilon)} .
$$

REMARK. If $w$ is a rational function then we can easily obtain better estimates than those in Lemma 1.

LEMMA 2. Let $w$ be an entire function of finite order $n \geq 1$ where $\lambda(w)<n$. Then there exists a constant $b \neq 0$ and there exists a set $E \subset(1, \infty)$ that has finite logarithmic measure, such that

$$
w^{\prime}(z) / w(z)=(1+o(1)) b z^{n-1}
$$

as $z \rightarrow \infty,|z| \notin E$. 
PrOOF. From the hypothesis and the Hadamard factorization theorem, it follows that $n$ is a positive integer and that $w(z)=h(z) \exp \left(c z^{n}\right)$ where $c \neq 0$ is some constant and $h(z)$ is an entire function that satisfies $\rho(h)<n$. Then

$$
w^{\prime}(z) / w(z)=h^{\prime}(z) / h(z)+c n z^{n-1} .
$$

Since $\rho(h)<n$, it follows from Lemma 1 (ii) that there exists a set $E \subset(1, \infty)$ that has finite logarithmic measure, such that

$$
\left|h^{\prime}(z) / h(z)\right|=o(1)|z|^{n-1}
$$

as $z \rightarrow \infty,|z| \notin E$. Combining (3.4) and (3.3) gives (3.2). This proves Lemma 2 .

Concerning Lemma 2, we mention Lemma 1 in [8].

LEMMA 3 [9]. Let $w$ be an entire function of order $\rho$ where $0<\rho<1 / 2$, and let $\varepsilon>0$ be a given constant. Then there exists a set $S \subset[0, \infty)$ that has upper density at least $1-2 \rho$ such that $|w(z)|>\exp \left(|z|^{\rho-\varepsilon}\right)$ for all $z$ satisfying $|z| \in S$.

For the definition of "upper density" in Lemma 3, see [9, p. 679].

LEMMA 4. Let $w$ be entire, and suppose that $\left|w^{\prime}(z)\right|$ is unbounded on some ray $\arg z=\theta$. Then there exists an infinite sequence of points $z_{n}=r_{n} e^{i \theta}$ where $r_{n} \rightarrow \infty$, such that $w^{\prime}\left(z_{n}\right) \rightarrow \infty$ and

$$
\left|w\left(z_{n}\right) / w^{\prime}\left(z_{n}\right)\right| \leq(1+o(1))\left|z_{n}\right|
$$

as $z_{n} \rightarrow \infty$.

ProOF. Let $M\left(r, w^{\prime}, \theta\right)=\max \left|w^{\prime}(z)\right|$ over all $z$ satisfying $0 \leq|z| \leq r$ and $\arg z=\theta$. It follows that there exists an infinite sequence of points $z_{n}=r_{n} e^{i \theta}$ where $r_{n} \rightarrow \infty$ such that $M\left(r_{n}, w^{\prime}, \theta\right)=\left|w^{\prime}\left(r_{n} e^{i \theta}\right)\right|$ for all $n$. Then for each $n$, we have

$$
\begin{aligned}
& w\left(z_{n}\right)=w(0)+\int_{0}^{z_{n}} w^{\prime}(z) d z \\
& \left|w\left(z_{n}\right)\right| \leq|w(0)|+r_{n}\left|w^{\prime}\left(z_{n}\right)\right| .
\end{aligned}
$$

Since $w^{\prime}\left(z_{n}\right) \rightarrow \infty$, we obtain (3.5).

LEMMA 5. Let $g(r)$ and $h(r)$ be monotone nondecreasing functions on $[0, \infty)$ such that $g(r) \leq h(r)$ for all $r \notin E \cup[0,1]$ where $E \subset(1, \infty)$ is a set of finite logarithmic measure. Let $\alpha>1$ be a given constant.

Then there exists an $r_{0}=r_{0}(\alpha)>0$ such that $g(r) \leq h(\alpha r)$ for all $r \geq r_{0}$.

ProOF. We will reason as on p. 519 of [2]. From the hypothesis on $E$, it follows that there exists an $r_{0}=r_{0}(\alpha)>0$, such that for any $r \geq r_{0}$, the interval $[r, \alpha r]$ must contain a point $r_{1}$ where $r_{1} \notin E \cup[0,1]$. Then $g(r) \leq g\left(r_{1}\right) \leq h\left(r_{1}\right) \leq h(\alpha r)$. This proves Lemma 5 .

LEMMA 6. Let $w$ be analytic on a ray $\arg z=\theta$, and suppose that for some constant $\alpha>1$ we have

$$
\left|w^{\prime}(z) / w(z)\right|=O\left(|z|^{-\alpha}\right)
$$

as $z \rightarrow \infty$ along $\arg z=\theta$. Then there exists a constant $c \neq 0$ such that $w(z) \rightarrow c$ as $z \rightarrow \infty$ along $\arg z=\theta$.

PROOF. From (3.6) it follows that there exists an $R_{0}>0$ and a simply connected domain $D$ such that $w^{\prime} / w$ is analytic on $D$ and where if $z$ satisfies $\arg z=\theta$ and 
$|z| \geq R_{0}$ then $z \in D$. Hence there exists an analytic function $F(z)$ on $D$ such that $F^{\prime}=w^{\prime} / w$ on $D$. If $z_{1}=r_{1} \exp (i \theta)$ and $z_{2}=r_{2} \exp (i \theta)$ are large, where $R_{0}<r_{1}<r_{2}$, then by consideration of

$$
F\left(z_{2}\right)-F\left(z_{1}\right)=\int_{z_{1}}^{z_{2}} \frac{w^{\prime}(\varsigma)}{w(\varsigma)} d \varsigma
$$

and (3.6), it can be deduced that there exists a constant $b$ such that $F(z) \rightarrow b$ as $z \rightarrow \infty$ along $\arg z=\theta$. It follows that there exists a constant $c \neq 0$ such that $w(z) \rightarrow c$ as $z \rightarrow \infty$ along $\arg z=\theta$.

4. Proof of Theorem 2. Suppose that $f \not \equiv 0$ is a solution of (2.1) where $\rho(f)<\infty$. From $(2.1)$ we have

$$
B(z)=-f^{\prime \prime} / f-A(z)\left(f^{\prime} / f\right) .
$$

Hence from Nevanlinna's fundamental estimate of the logarithmic derivative, we obtain that $m(r, B) \leq m(r, A)+O(\log r)$ as $r \rightarrow \infty$. Q.E.D.

5. Proof of Theorem 1. Suppose that $f \not \equiv 0$ is a solution of equation (2.1) where $\rho(f)<\infty$. We will assume that $\lambda(f)<\rho(f)$.

Set $n=\rho(f)$. Then $n \geq 1$ is an integer. From (2.1),

$$
A(z)=-B(z)\left(f / f^{\prime}\right)-f^{\prime \prime} / f^{\prime}
$$

From Lemma 2, there exists a constant $b \neq 0$ and a set $E \subset(1, \infty)$ that has finite logarithmic measure, such that

$$
f^{\prime}(z) / f(z)=(1+o(1)) b z^{n-1}
$$

as $z \rightarrow \infty,|z| \notin E$. From (5.2), (5.1), and the fundamental estimate of the logarithmic derivative, we obtain

$$
m(r, A) \leq m(r, B)+O(\log r)
$$

as $r \rightarrow \infty, r \notin E$. Since $T(r, B) \leq T(r, A)+O(\log r)$ as $r \rightarrow \infty$ from (2.3), we obtain that

$$
T(r, A)=T(r, B)+O(\log r)
$$

as $r \rightarrow \infty, r \notin E$.

First suppose that $B(z)$ is transcendental. Then $A(z)$ is transcendental from (5.3). Since the Nevanlinna characteristic is a nondecreasing function of $r$, we can deduce from (5.3) and Lemma 5 that $\rho(A)=\rho(B)$. This is case (ii).

Now suppose that $B(z) \not \equiv 0$ is a polynomial. Then $A(z)$ is a polynomial from (5.3). We will use the well-known transformation

$$
w(z)=f(z) \exp \left(\frac{1}{2} \int A(z) d z\right)
$$

which, when substituted into (2.1), gives

$$
w^{\prime \prime}+G(z) w=0
$$

where $G(z)=B(z)-(A(z))^{2} / 4-A^{\prime}(z) / 2$. If $G(z) \not \equiv 0$, then (see [4, Theorem 1])

$$
\rho(w)=1+\operatorname{deg}(G) / 2 .
$$


Since $\lambda(f)<\rho(f)$, we also have that

$$
f(z)=h(z) e^{Q(z)}
$$

where $h(z)$ is either a canonical product or $h(z) / z$ is a canonical product, $Q(z)$ is a polynomial, and $\rho(h)=\lambda(f)<\rho(f)=\operatorname{deg}(Q)$. Substitution of (5.7) into (2.1) gives

$$
h^{\prime \prime} / h+\left(2 Q^{\prime}+A\right) h^{\prime} / h+Q^{\prime \prime}+\left(Q^{\prime}\right)^{2}+A Q^{\prime}+B=0 .
$$

For convenience, we set $\alpha=\operatorname{deg}(A)$ and $\beta=\operatorname{deg}(B)$.

First suppose that $\beta>2 \alpha$. Then by applying the Wiman-Valiron theory [23] to equation (2.1), we obtain that $\rho(f)=1+\beta / 2$. Thus $\rho(w)=\rho(f)$ from (5.6). Since from (5.4), $\lambda(w)=\lambda(f)<\rho(f)=\rho(w)$, it follows from (5.5) and [4, Theorem 1] that $w$ has only finitely many zeros. Hence $f$ has only finitely many zeros. This is case (iv)(a).

Now suppose that $\beta<2 \alpha$. Then by applying the Wiman-Valiron theory to equation (2.1), we obtain that $\rho(f) \leq 1+\alpha$. From (5.6), $\rho(w)=1+\alpha$. Thus $\rho(w) \geq \rho(f)>\lambda(f)=\lambda(w)$, and so once again we obtain from (5.5) and [4, Theorem 1] that $w$ and $f$ each have only finitely many zeros. If $\rho(f)=1+\alpha$, then we obtain case (iv)(d). Now suppose $\rho(f)<1+\alpha$. From (5.7), $\operatorname{deg}\left(Q^{\prime}\right)=$ $\rho(f)-1<\alpha$, and $h$ is a polynomial. Then by letting $z \rightarrow \infty$ in (5.8), we deduce that $\operatorname{deg}\left(A Q^{\prime}\right)=\operatorname{deg}(B)$. This yields $\alpha+\rho(f)-1=\beta$. This is case (iv)(c).

Now suppose that $\beta=2 \alpha=\operatorname{deg}\left(4 B-A^{2}\right)$ and $G(z) \equiv 0$ in (5.5). Then $A(z) \not \equiv 0$ (since $B(z) \not \equiv 0)$. Then from (5.5) and (5.4), it follows that $f$ has only finitely many zeros and that $\rho(f)=1+\alpha$. This is case (iv)(b).

Next suppose that $\beta=2 \alpha=\operatorname{deg}\left(4 B-A^{2}\right)$ and $G(z) \not \equiv 0$. Then $\rho(w)=1+\alpha$ from (5.6). By applying the Wiman-Valiron theory to (2.1), we get $\rho(f) \leq 1+\alpha$. Thus $\rho(w) \geq \rho(f)>\lambda(f)=\lambda(w)$, and so (as above) $w$ and $f$ each have only finitely many zeros. If $\rho(f)=1+\alpha$ then we get case (iv)(b). If $\rho(f)<1+\alpha$, then as above we can deduce from (5.7) and (5.8) that $\operatorname{deg}\left(Q^{\prime}\right)=\rho(f)-1<\alpha, h$ is a polynomial, and $\operatorname{deg}\left(A Q^{\prime}\right)=\operatorname{deg}(B)$, which yields $\alpha+\rho(f)-1=\beta$. But this contradicts $\beta=2 \alpha$ and $\rho(f)<1+\alpha$.

Last, we assume that $\beta=2 \alpha>\operatorname{deg}\left(4 B-A^{2}\right)$. Then $\rho(w)<1+\alpha$ from (5.6) and (5.5). Then from (5.4), we obtain that $\rho(f)=1+\alpha$. If $f$ has only finitely many zeros then we have case (iv)(b). If $f$ has infinitely many zeros, then from (5.4), (5.5), (5.6), and [4, Theorem 1] we deduce that $\lambda(f)=\lambda(w)=\rho(w) \geq 1$. This is case (iii).

The proof of Theorem 1 is now complete.

6. Proof of Theorem 3. Set $\mu=\rho(f)$. From Lemma 1(i), there exists a set $E \subset[0,2 \pi)$ that has linear measure zero, such that if $\psi_{0} \in[0,2 \pi)-E$, then

$$
\left|f^{\prime \prime}(z) / f^{\prime}(z)\right|=o(1)|z|^{\mu}
$$

as $z \rightarrow \infty$ along $\arg z=\psi_{0}$.

Now suppose that $\left|f^{\prime}(z)\right|$ is unbounded on some ray $\arg z=\phi_{0}$ where $\phi_{0} \in$ $\left[\theta_{1}, \theta_{2}\right]-E$. Then from Lemma 4 , there exists an infinite sequence of points $z_{n}=$ $r_{n} \exp \left(i \phi_{0}\right)$ where $r_{n} \rightarrow \infty$, such that $f^{\prime}\left(z_{n}\right) \rightarrow \infty$ and

$$
\left|f\left(z_{n}\right) / f^{\prime}\left(z_{n}\right)\right| \leq(1+o(1))\left|z_{n}\right|
$$


as $z_{n} \rightarrow \infty$. From (2.1),

$$
|A(z)| \leq\left|f^{\prime \prime} / f^{\prime}\right|+|B(z)|\left|f / f^{\prime}\right| .
$$

By using (6.2), (6.1), (2.4), and (2.5), we will obtain a contradiction in (6.3) as $z_{n} \rightarrow \infty$. Therefore, $\left|f^{\prime}(z)\right|$ is bounded on any ray $\arg z=\phi$ where $\phi \in\left[\theta_{1}, \theta_{2}\right]-E$. It then follows from the classical Phragmén-Lindelöf theorem [18, p. 214] that there exists a constant $M>0$ such that

$$
\left|f^{\prime}(z)\right| \leq M
$$

for all $z \in S(\varepsilon)$.

If $\theta_{0} \in\left[\theta_{1}+\varepsilon, \theta_{2}-\varepsilon\right]-E$, then when $\arg z=\theta_{0}$, we obtain from (6.4) that

$$
|f(z)| \leq|f(0)|+\left|\int_{0}^{z} f^{\prime}(u) d u\right| \leq|f(0)|+M|z| .
$$

From (6.5), (6.1), and (2.1), we obtain that

$$
|A(z)|\left|f^{\prime}(z)\right| \leq o(1)\left|f^{\prime}(z)\right||z|^{\mu}+|B(z)|\{|f(0)|+M|z|\}
$$

as $z \rightarrow \infty$ along $\arg z=\theta_{0}$. From (6.6), (2.4), and (2.5), we can deduce that

$$
\left|f^{\prime}(z)\right| \leq \exp \left\{-(1+o(1)) \alpha|z|^{\beta}\right\}
$$

as $z \rightarrow \infty$ along $\arg z=\theta_{0}$. By using an application of the Phragmén-Lindelöf theorem on (6.7), it can be deduced that

$$
\left|f^{\prime}(z)\right| \leq \exp \left\{-(1+o(1)) \alpha|z|^{\beta}\right\}
$$

as $z \rightarrow \infty$ in $S(2 \varepsilon)$. This gives $k=1$ in (2.7).

Now let $z \in S(3 \varepsilon)$ where $|z|>1$, let $\gamma$ be a circle of radius one with center at $z$, and let $k \geq 1$ be an integer. Then from the Cauchy integral formula and (6.8), we obtain as $z \rightarrow \infty$ in $S(3 \varepsilon)$,

$$
\left|f^{(k)}(z)\right| \leq \frac{(k-1) !}{2 \pi} \int_{\gamma} \frac{\left|f^{\prime}(u)\right|}{|u-z|^{k}}|d u| \leq \exp \left\{-(1+o(1)) \alpha|z|^{\beta}\right\} .
$$

This proves (2.7).

Now fix $\theta$ where $\theta_{1}+\varepsilon \leq \theta \leq \theta_{2}-\varepsilon$, and set

$$
a_{0}=\int_{0}^{\infty} f^{\prime}\left(t e^{i \theta}\right) e^{i \theta} d t
$$

where we note that $a_{0} \in \mathbf{C}$ from (2.7). Let $z=|z| e^{i \psi}$ where $\theta_{1}+\varepsilon \leq \psi \leq \theta_{2}-\varepsilon$. Then from Cauchy's theorem and (6.9) we obtain

$$
\begin{aligned}
f(z)-f(0)-a_{0} & =\int_{0}^{z} f^{\prime}(u) d u-\int_{0}^{\infty} f^{\prime}\left(t e^{i \theta}\right) e^{i \theta} d t \\
& =\int_{\theta}^{\psi} f^{\prime}\left(|z| e^{i \xi}\right) i|z| e^{i \xi} d \xi-\int_{|z|}^{\infty} f^{\prime}\left(t e^{i \theta}\right) e^{i \theta} d t .
\end{aligned}
$$

From (6.10) and (2.7), it can be deduced that

$$
|f(z)-b| \leq \exp \left\{-(1+o(1)) \alpha|z|^{\beta}\right\}
$$


as $z \rightarrow \infty$ in $S(\varepsilon)$, where $b=f(0)+a_{0}$. (Note: It follows that $a_{0}$ in (6.9) is independent of $\theta$.) Since (6.11) is the inequality (2.6), it remains only to show that $b \neq 0$.

From Lemma $1(\mathrm{i})$, there exists a ray $\arg z=\psi_{1}$ where $\theta_{1}+\varepsilon \leq \psi_{1} \leq \theta_{2}-\varepsilon$, such that

$$
\left|f^{\prime \prime}(z) / f(z)\right|=o(1)|z|^{2 \mu}
$$

as $z \rightarrow \infty$ along $\arg z=\psi_{1}$ (where $\mu=\rho(f)$ ). Then from (6.12), (2.4), (2.5), and (2.1), we obtain that

$$
\left|\frac{f^{\prime}(z)}{f(z)}\right| \leq\left|\frac{B(z)}{A(z)}\right|+\frac{\left|f^{\prime \prime}(z)\right|}{|f(z) A(z)|} \leq \exp \left\{-(1+o(1)) \alpha|z|^{\beta}\right\}
$$

as $z \rightarrow \infty$ along $\arg z=\psi_{1}$. By applying Lemma 6 to (6.13), and noting that $f(z) \rightarrow b$ as $z \rightarrow \infty$ in $S(\varepsilon)$ from (6.11), we see that $b \neq 0$.

Thus part (i) is proved, and the proof of Theorem 3 is now complete.

7. Proof of Theorem 5. Suppose that $f \not \equiv 0$ is a solution of equation (2.1) where $\rho(f)<\infty$. Let $\varepsilon>0,\left\{\phi_{k}\right\}$, and $\left\{\theta_{k}\right\}$ be as in the hypothesis. From (2.13) and $\rho(B)<\beta$, it follows from Theorem 3(i) that $|f(z)|$ is bounded in each angle $\phi_{k}+\varepsilon \leq \arg z \leq \theta_{k}-\varepsilon(k=1,2, \ldots, n)$. Since $\varepsilon$ can be arbitrarily small, it follows from (2.12) and the Phragmén-Lindelöf theorem that $|f(z)|$ is bounded in the whole finite plane. Thus $f$ is a nonzero constant from Liouville's theorem, and this contradicts (2.1).

8. Proof of Theorem 4. Suppose that $f \not \equiv 0$ is a solution of equation (2.1) where $\rho(f)<\infty$. Set $\mu=\rho(f)$. Then from Lemma 1(i), there exists a real constant $\psi_{0}$ where $\theta_{1} \leq \psi_{0} \leq \theta_{2}$, such that

$$
\left|f^{\prime \prime}(z) / f(z)\right|=o(1)|z|^{2 \mu} \quad \text { and } \quad\left|f^{\prime}(z) / f(z)\right|=o(1)|z|^{\mu}
$$

as $z \rightarrow \infty$ along $\arg z=\psi_{0}$. Then from (8.1) and (2.1) we obtain that

$$
|B(z)| \leq o(1)|z|^{\mu}|A(z)|+o(1)|z|^{2 \mu}
$$

as $z \rightarrow \infty$ along $\arg z=\psi_{0}$, and this contradicts (2.10) and (2.11).

9. Proof of Theorem 6. Let $f \not \equiv 0$ be a solution of equation (2.1). If $A(z)$ is transcendental with $\rho(A)<1 / 2$ and $B(z)$ is a polynomial, then Ozawa [19, Theorem 1] showed that $\rho(f)=\infty$.

Now suppose that $\rho(f)<\infty$ and $\rho(B)<\rho(A)<1 / 2$. We assume first that $f$ has an infinite number of zeros. From integration of $f^{\prime} / f$ around circles $|z|=r$, it follows from the residue theorem that there exists a constant $R>0$ such that for each $r>R$ there must be a point $z_{r}$ that satisfies $\left|z_{r}\right|=r$ and

$$
\left|f^{\prime}\left(z_{r}\right) / f\left(z_{r}\right)\right|>1 /\left|z_{r}\right| \text {. }
$$

Now let $\mu$ be a constant that satisfies $\rho(B)<\mu<\rho(A)$. Then from Lemma 3, there exists a set $S \subset[0, \infty)$ that has positive upper density such that

$$
|A(z)|>\exp \left(|z|^{\mu}\right)
$$

for all $z$ satisfying $|z| \in S$. (For the definition of "upper density", see [9, p. 679]). Also, by Lemma 1 (iii), there exists a set $E \subset[0, \infty)$ that has finite linear measure, 
such that for all $z$ satisfying $|z| \notin E$ we have

$$
\left|f^{\prime \prime}(z) / f(z)\right| \leq|z|^{\alpha}
$$

where $\alpha=2 \rho(f)+1$.

Using (2.1), (9.1), (9.2), and (9.3), we see that there exist arbitrarily large $z$ such that

$$
\frac{\exp \left(|z|^{\mu}\right)}{|z|}<\left.|A(z)| \frac{f^{\prime}(z)}{f(z)}|\leq| B(z)|+| \frac{f^{\prime \prime}(z)}{f(z)}|\leq| B(z)|+| z\right|^{\alpha},
$$

and this contradicts that $\rho(B)<\mu$.

Next we assume that $f$ has a finite number of zeros. Then $f=P e^{Q}$ where $P \not \equiv 0$ and $Q$ are polynomials. Substituting this into $(2.1)$ gives

$$
P^{\prime \prime}+2 Q^{\prime} P^{\prime}+Q^{\prime \prime} P+\left(Q^{\prime}\right)^{2} P+A(z)\left(P^{\prime}+Q^{\prime} P\right)+B(z) P=0 .
$$

Since $\rho(B)<\rho(A)$, it follows from (9.4) that $P^{\prime}+Q^{\prime} P \equiv 0$. Thus $Q^{\prime} \equiv 0$ and $P^{\prime} \equiv 0$. Then $f$ is a nonzero constant which contradicts $(2.1)$.

Thus it is impossible to have $\rho(f)<\infty$ and $\rho(B)<\rho(A)<1 / 2$. The proof of Theorem 6 is now complete.

10. Proof of Theorem 7. Suppose that $f \not \equiv 0$ is a solution of equation (2.1) where $\rho(f)<\infty$. Set $\beta=\rho(f)$. From Lemma $1(\mathrm{i})$, there exists a set $E \subset[0,2 \pi)$ that has linear measure zero, such that if $\psi_{0} \in\left[\phi_{k}, \theta_{k}\right]-E$ for some $k$, then

$$
\left|f^{\prime}(z) / f(z)\right|=O\left(|z|^{\beta}\right) \text { and }\left|f^{\prime \prime}(z) / f(z)\right|=O\left(|z|^{2 \beta}\right)
$$

as $z \rightarrow \infty$ along $\arg z=\psi_{0}$. From (10.1), (2.16), and (2.1), we obtain that

$$
|B(z)| \leq|A(z)|\left|f^{\prime}(z) / f(z)\right|+\left|f^{\prime \prime}(z) / f(z)\right|=O\left(|z|^{\sigma}\right)
$$

as $z \rightarrow \infty$ along $\arg z=\psi_{0}$, where $\sigma=\alpha+2 \beta$. Now let $\varepsilon>0$ be a small constant that satisfies $\rho(B)<\pi /(\mu+2 \varepsilon$ ) (this is possible since $\rho(B)<\pi / \mu$ ). By using the Phragmén-Lindelöf theorem on (10.2), it can be deduced that for some integer $m>0$,

$$
|B(z)|=O\left(|z|^{m}\right)
$$

as $z \rightarrow \infty$ in $\phi_{k}+\varepsilon \leq \arg z \leq \theta_{k}-\varepsilon$ for $k=1, \ldots, n$. Now for each $k$, we have from (2.15) that $\phi_{k+1}+\varepsilon-\left(\theta_{k}-\varepsilon\right) \leq \mu+2 \varepsilon$, and so $\rho(B)<\pi /\left(\phi_{k+1}-\theta_{k}+2 \varepsilon\right)$. Hence from using the Phragmén-Lindelöf theorem on (10.3), we can deduce that $|B(z)|=O\left(|z|^{m}\right)$ as $z \rightarrow \infty$ in the whole complex plane. This means that $B(z)$ is a polynomial which contradicts our hypothesis. This proves Theorem 7 .

11. More examples. We will now give some more examples which will illustrate some possibilities that can occur and which will also exhibit the sharpness of some of our results.

EXAMPLE 1. Let $Q$ be any nonconstant polynomial, let $B \not \equiv 0$ be any entire function with $\rho(B)<\operatorname{deg}(Q)$, let $f$ be any antiderivative of $e^{Q}$ that satisfies $\lambda(f)=$ $\operatorname{deg}(Q)$, and set $A=-Q^{\prime}-B f e^{-Q}$. Then $\rho(B)<\rho(A)=\operatorname{deg}(Q)=\rho(f)$, and $f^{\prime \prime}+A(z) f^{\prime}+B(z) f=0$.

This shows that it is possible to have a finite order solution $f \not \equiv 0$ of an equation of the form $(2.1)$ where $\rho(B)<\rho(A)$ and where $\rho(A)$ may be any positive integer. In contrast, we have Theorem 6 .

EXAMPLE 2 . Let $P$ be a polynomial with degree $\geq 2$, such that $P^{\prime}$ has only simple zeros, and where $P^{\prime}(z) \neq 0$ whenever $\exp (-P(z))=1$. Let $Q$ be a polynomial 
such that

$$
Q(z)=\frac{P^{\prime \prime}(z)}{e^{-P(z)}-1}
$$

whenever $P^{\prime}(z)=0$. Then $Q \not \equiv 0$. For example, $Q$ can be Lagrange's interpolation polynomial where $\operatorname{deg}(Q) \leq \operatorname{deg}(P)-2$ (see [18, pp. 67-71]). Set

$$
A=\frac{Q\left(e^{-P}-1\right)-P^{\prime \prime}-\left(P^{\prime}\right)^{2}}{P^{\prime}}
$$

and set $f=e^{P}-1$. Then $A$ is entire, $\rho(A)=\operatorname{deg}(P)=\rho(f)$, and

$$
f^{\prime \prime}+A(z) f^{\prime}+Q(z) f=0 \text {. }
$$

This gives another example like Example 1 (i.e. where " $\rho(B)<\rho(A)$ " and ...), and it also gives an illustration of Theorem 3 where the inequalities (2.6) and (2.7) are both sharp in the sense that neither of the two numbers " $\alpha$ " or " $\beta$ " in either (2.6) or (2.7) could be replaced by a larger number.

EXAMPLE 3. Let $\alpha$ be any real number that satisfies $0 \leq \alpha \leq 1$. We will show that there exist three transcendental entire functions $f, A, B$, all of finite order, such that equation (2.1) holds, and where $\delta(0, f)=\alpha$.

Equation (2.2) easily gives the assertion when $\alpha=1$.

Now suppose that $\alpha<1$. Set $\beta=(1-\alpha)^{-1}$. It can be verified that $f(z)=$ $\exp (\beta z)+\exp ((\beta-1) z)$ satisfies the equation

$$
f^{\prime \prime}+\left(e^{\beta z}+e^{(\beta-1) z}+1-2 \beta\right) f^{\prime}+\left(\beta^{2}-\beta+(1-\beta) e^{(\beta-1) z}-\beta e^{\beta z}\right) f=0 .
$$

We have that $N(r, 0, f)=(1+o(1)) r / \pi$ as $r \rightarrow \infty$. Since $\beta \geq 1$, it can be found that $T(r, f)=(1+o(1)) \beta r / \pi$ as $r \rightarrow \infty$. Therefore $\delta(0, f)=\alpha$.

The assertion is proved.

EXAMPLE 4. We will now construct a general example of case (iii) in Theorem 1. Let $Q(z)$ be any nonconstant polynomial. Then [4, Theorem 1] there exists a solution $w \not \equiv 0$ of the equation

$$
w^{\prime \prime}-\frac{Q^{\prime}(z)}{2} w=0
$$

that satisfies $\lambda(w)=\rho(w)=(1+\operatorname{deg}(Q)) / 2$. Then $f(z)=w(z) \exp \left(-\frac{1}{2} \int Q(z) d z\right)$ satisfies the equation

$$
f^{\prime \prime}+Q(z) f^{\prime}+\frac{(Q(z))^{2}}{4} f=0
$$

and $1 \leq \lambda(f)=(1+\operatorname{deg}(Q)) / 2<\rho(f)=1+\operatorname{deg}(Q)$.

Furthermore, if equation (11.1) possesses two linearly independent solutions $w_{1}$ and $w_{2}$ where $\lambda\left(w_{i}\right)=\rho\left(w_{i}\right)=(1+\operatorname{deg}(Q)) / 2$ for $i=1,2$, then equation (11.2) will possess two linearly independent solutions $f_{1}$ and $f_{2}$ where $1 \leq \lambda\left(f_{i}\right)<\rho\left(f_{i}\right)$ for 
$i=1,2$. For example, when $Q(z)=2 z$, we obtain that $f_{1}(z)=(\sinh z) \exp \left(-z^{2} / 2\right)$ and $f_{2}(z)=(\cosh z) \exp \left(-z^{2} / 2\right)$ both satisfy the equation $f^{\prime \prime}+2 z f^{\prime}+z^{2} f=0$.

EXAMPLE 5. We now give examples of each of the four situations (a), (b), (c), and (d) in Theorem 1 (iv).

If $P$ is a polynomial of degree $n \geq 1$, then $f=e^{P}$ satisfies equation (2.1) where $A \equiv 0$ and $B=-P^{\prime \prime}-\left(P^{\prime}\right)^{2}$. When $n \geq 2$, we have $2 \operatorname{deg}(A)=0<\operatorname{deg}(B)=$ $2 n-2$ and $\rho(f)=n=1+(\operatorname{deg}(B)) / 2$, which is case (a). When $n=1$, we have $2 \operatorname{deg}(A)=\operatorname{deg}(B)=0$ and $\rho(f)=1=1+\operatorname{deg}(A)$, which is case (b).

Let $k \geq 1$ be an integer. Then $f(z)=\exp \left(z^{k}\right)$ satisfies equation (2.2) where $H(z)=z^{k}$, and this is an example of case (c).

Let $k \geq 2$ be an integer. Then $f(z)=\exp \left(z^{k}\right)$ satisfies equation (2.2) where $H(z) \equiv 0$, and this is an example of case (d).

\section{REFERENCES}

1. I. Amemiya and M. Ozawa, Non-existence of finite order solutions of $w^{\prime \prime}+e^{-z} w^{\prime}+Q(z) w=0$, Hokkaido Math. J. 10 (1981), 1-17.

2. S. B. Bank, On the value distribution theory for entire solutions of second-order linear differential equations, Proc. London Math. Soc. (3) 50 (1985), 505-534.

3. _ Three results in the value-distribution theory of solutions of linear differential equations, Kodai Math. J. 9 (1986), 225-240.

4. S. B. Bank and I. Laine, On the oscillation theory of $f^{\prime \prime}+A f=0$ where $A$ is entire, Trans. Amer. Math. Soc. 273 (1982), 351-363.

5. __ Representations of solutions of periodic second order linear differential equations, J. Reine Angew. Math. 344 (1983), 1-21.

6. __ On the zeros of meromorphic solutions of second-order linear differential equations, Comment. Math. Helv. 58 (1983), 656-677.

7. S. B. Bank, I. Laine, and J. K. Langley, On the frequency of zeros of solutions of second order linear differential equations, Resultate Math. 10 (1986), 8-24.

8. S. B. Bank and J. K. Langley, On the oscillation of solutions of certain linear differential equations in the complex domain, Proc. Edinburgh Math. Soc. (to appear).

9. A. S. Besicovitch, On integral functions of order < 1, Math. Ann. 97 (1927), 677-695.

10. M. L. Cartwright, Integral functions, Cambridge Univ. Press, London, 1962.

11. M. Frei, Sur l'ordre des solutions entière d'une équation différentielle linéaire, C. R. Acad. Sci. Paris 236 (1953), 38-40.

12. __ Über die subnormalen Lösungen der Differentialgleichung $w^{\prime \prime}+e^{-z} w^{\prime}+($ Konst.) $w=0$, Comment. Math. Helv. 36 (1961), 1-8.

13. G. G. Gundersen, On the question of whether $f^{\prime \prime}+e^{-z} f^{\prime}+B(z) f=0$ can admit a solution $f \not \equiv 0$ of finite order, Proc. Roy. Soc. Edinburgh 102A (1986), 9-17.

14. __ Estimates for the logarithmic derivative of a meromorphic function, plus similar estimates, J. London Math. Soc. (to appear).

15. W. K. Hayman, Meromorphic functions, Clarendon Press, Oxford, 1964.

16. E. Hille, Ordinary differential equations in the complex domain, Wiley, New York, 1976.

17. J. K. Langley, On complex oscillation and a problem of Ozawa, Kodai Math. J. 9 (1986), 430-439.

18. A. I. Markushevich, Theory of functions of a complex variable, Vol. II, translated by R. A. Silverman, Prentice-Hall, Englewood Cliffs, N.J., 1965.

19. M. Ozawa, On a solution of $w^{\prime \prime}+e^{-z} w^{\prime}+(a z+b) w=0$, Kodai Math. J. 3 (1980), 295309.

20. K. Pöschl, Zur Frage des Maximalbetrages der Lösungen linearer Differentialgleichungen zweiter Ordnung mit Polynomkoeffizienten, Math. Ann. 125 (1953), 344-349.

21. J. Rossi, Second order differential equations with transcendental coefficients, Proc. Amer. Math. Soc. 97 (1986), 61-66. 
22. L.-C. Shen, Solution to a problem of $S$. Bank regarding the exponent of convergence of the zeros of the solutions of the differential equation $f^{\prime \prime}+A f=0$, Kexue Tongbao (English ed.) 30 (1985), 1579-1585.

23. G. Valiron, Lectures on the general theory of integral functions, translated by E. F. Collingwood, Chelsea, New York, 1949.

24. H. Wittich, Neuere Untersuchungen über eindeutige analytische Funktionen, Springer-Verlag, Berlin, 1955.

25. __ Subnormale Lösungen der Differentialgleichung $w^{\prime \prime}+p\left(e^{z}\right) w^{\prime}+q\left(e^{z}\right) w=0$, Nagoya Math. J. 30 (1967), 29-37.

Department of Mathematics, UNiversity of NeW Orleans, NeW ORLEANS, LOUISIANA 70148 